\title{
Research and Mathematic Simulation of the Pollution Particle Motion Process in the Fluid Stream under Effluents Treatment
}

\author{
Jamil Safarov ${ }^{1, *}$ \\ ${ }^{1}$ Azerbaijan State Agricultural University, Ganja, 262, Ataturk Avenue, Azerbaijan. AZ2000
}

\begin{abstract}
The article covers the issues of the mathematic simulation of the pollution particle motion process in the fluid stream, and there are defined the options influencing the process of the sweat. There are also analysed the challenging problems of the effluent water dispose. There is also devoted attention to the updating of the sedimentation process by the use of constrained features which exclude sediments in the objectionable points of the cleansing system and etc.
\end{abstract}

\section{Introduction}

The modern industrial development and the environment pollution coming from it, a number of large-scale of the technological accidents force to highlight the ecological problems last years; the ecological problems solution is hardwired with the human survival [1]. One of the main problems in this situation is cleaning-out of the disposed effluent water. As it is known their under treatment results in accumulation of the residual impurity in the water reservoirs and rivers which causes the strong difficulties in the further use of these water reservoirs in the economic operations.

The most refractory components which are hard to be excluded from the effluent waters are the lubricating mineral oils and surface-active substances (SAS). The named components form the basis of the lubricatingcooling fluid (LCF) used while mechanical metalworking. For this reason the effluents treatment at the machine builders containing significant amount of the exhausted lubricating-cooling fluid is related to the great technical difficulties and serious material expenses.

The problem is also complicated by means of equipment absence in present time - the equipment allowing conducting the effluents treatment works more effectively [1-5].

In conjunction with high tenacity of the dispersoid solution and flimsy biological oxidizability of the lubricating mineral oils such kind of waters fall for the mechanical, physico-chemical and biological methods of the cleaning out very bad. Besides, dispersoid solutions entitled to the cleaning out are characterized by the high level of adhesiveness to the operating device of the processing equipment, which demands the implementation of the special equipment, including the vibrational one.

Insufficient level of cleaning out of the effluent waters containing the exhausted lubricating-cooling fluid causes their accumulation and long-term conservation in the water and ground.

It is obvious that the reduction of dispersion medium fraction in spill water, including the dispersion solution is the urgent problem, the solution of which can significantly influence the improvement of the ecological setting.

Recently while effluents treatment the vibrational technical processes are spread together with the magnetically separation and coagulation. The attractiveness of such kind of clearing out technologies can be explained by their generality, processibillity, automatization ability of the cleaning out process by quite simple methods, and also the concomitant cleaning out. So the issues of research in the laboratory and industrial conditions of the cleaning process and also the development, field research and implementation of the cleaning out machines are currently central and are of great national economic importance.

Some of the main processes defining the quality of the mechanical cleaning out of the fluid are the sedimentation processes, the effectiveness of which can be increased by implementation of the compounding technologies, magnetically coagulation and separation, transportation and filtration. These technological processes can be realized by implementation of the equipment developed on the basis of the vibrational machines, realizing the optimized performances of the end-effector movement in accordance with the technological criteria of the quality $[5,6]$.

At the same time the further development and implementation of the vibrational technologies into the cleaning out processes is constrained by absence of the developed theory of the specialized vibrational equipment which can be implemented for the work with dispersoid solutions, sludge and the other sizy dispersion mediums.

Feed waters are the polydisperse systems because at the same time they can contain particles the size of 
which fit the size of the colloidal and gross disperse particles.

Circuit schematics of wastewater technology

In actual fact circuit schematics of wastewater technology is focused on organization of transit of the feed water from the driving point to the way out; in such a case the suppression of the shot on the predetermined ground must happen on the trace. The ultimate particles which cannot be sooted in virtue of time limitation of the technological process have to be either filtered out or increased in the amount by curdling $[1,3,7]$.

Let us consider the process flowsheet of the effluents treatment from the pollution, which contains from three main zones (pic.1): mixing, suppression and filtration.

In the first zone the effluent water are affected by vibrational activation dopants - facilities creating the vibrational mixing and turbulization of the fluid, which allows to fully exclude the process of impaction of particles and formation of the sediments on the useful area of the pipes and processing equipment.

Depending on nature and mode of behavior of the fallout under the vibrational impact there can be defined three physical models: free of distortion, elastoviscous deformed and viscoplastic thixotrop; thus under the terrestrial gravity equitable in size with the adhesion force, it is preferentially to dispatch the extension vibration to the filter surface.

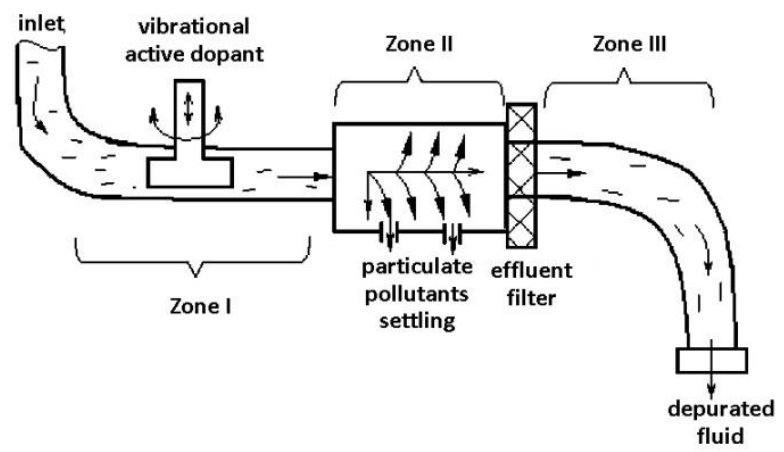

Fig.1. Process flow sheet of the effluents treatment

The function of the vibrational impact in the issue under consideration is the limit of stretching strain and plastic consistency of the settlement, the amounts of which come down with increase of the vibrations intension [2].

In the second zone the hindered settling of the particulate pollutant from the fluid takes place by the action of gravitational force for account of minimization of the fluid flow rate which allows realizing the guaranteed extraction of particles from the flux and their further utilization.

In the third zone the after purification of sewage water and movement of the depurated fluid to the terrestrial environment or to the secondary use take place. By the 'after purification' term it is meant the methods and processes replenishing the traditional process flow sheet of the effluents treatment of the set composition, at that any necessary and reachable removal or decomposition rate of the pollution is possible during the process of after purification.

\subsection{Research of the unsteady motion of the fluid in the cylinder bore of the settlement space of the particles from fluid.}

By way of the model which describes the fluid motion in the slug catcher we will choose the noncontractible treacle. For it $\mu=$ const and $\rho=$ const (pic. 2).

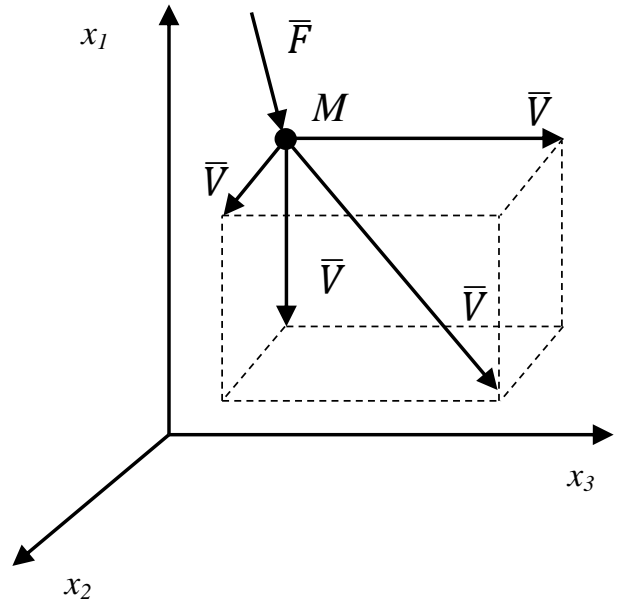

Fig. 2. Resultant vector dispatch of the local velocity of the fluid $\mathrm{M}$ on the coordinate axis

We will consider the nonstationary flow of the fluid, which is characterized by variability of velocity and pressure field during time (also referred to as unstable motion), arising under the accelerated or sluggish fluid motion or dosing control valve [8].

For the derivation of differential equations of motion of the ductile incompressible liquid we use the equations of continua motion on the ropes in the form of Eulerian equation [8-10]:

$$
\frac{d \bar{V}}{d t}=\frac{\partial \bar{V}}{\partial t}+(\bar{V} \cdot \nabla) \bar{V}=\bar{F}-\frac{1}{\rho}
$$

$\operatorname{grad}$;

$$
\operatorname{div} \mathrm{V}=0 \text {, }
$$

where $=(\mathrm{V} 1, \mathrm{~V} 2, \mathrm{~V} 3) \mathrm{T},=(\mathrm{F} 1, \mathrm{~F} 2, \mathrm{~F} 3) \mathrm{T}$ - velocity vector and reduced vector of volume force; $\mathrm{Vi}=(\mathrm{x \kappa}, \mathrm{t})$, $\mathrm{Fi}$ - projection of the speed and volume force onto the relevant reference axis (i, $\kappa=1,2,3) ; \rho$ - fluid density; $p$ - hydrodynamic pressure or the pressure in the given flow point.

The stress tensor is written as

$$
\mathrm{P}=\left(\begin{array}{lll}
\mathrm{p}_{11} & \mathrm{p}_{12} & \mathrm{p}_{13} \\
\mathrm{p}_{21} & \mathrm{p}_{22} & \mathrm{p}_{23} \\
\mathrm{p}_{31} & \mathrm{p}_{32} & \mathrm{p}_{33}
\end{array}\right)
$$

where $\mathrm{p}_{11}, \mathrm{p}_{22}, \mathrm{p}_{33},-$ are direct stress, and $\mathrm{p}_{12}, \mathrm{p}_{23}$, $p_{13}$ - tangential stress, enclosed to the normal to each other areas in the given environment.

Let us write down the Navier-Stokes equations for the Newtonian incompressible of the treacle $[8,10]$ :

$$
\begin{gathered}
\frac{d V_{1}}{d t}=F_{1}-\frac{1}{\rho} \frac{\partial p}{\partial x_{1}}+v \nabla^{2} V_{1} \\
\frac{d V_{2}}{d t}=F_{2}-\frac{1}{\rho} \frac{\partial p}{\partial x_{2}}+v \nabla^{2} V_{2}
\end{gathered}
$$




$$
\begin{gathered}
\frac{d V_{2}}{d t}=F_{3}-\frac{1}{\rho} \frac{\partial p}{\partial x_{3}}+v \nabla^{2} V_{13}, \\
\text { or shotly }
\end{gathered}, \begin{gathered}
\frac{d V_{i}}{d t}=F_{i}-\frac{1}{\rho} \frac{\partial p}{\partial x_{i}}+v \nabla^{2} V_{i}, \\
\frac{d V_{i}}{d x_{k}}=0(i, k=1,2,3),
\end{gathered}
$$

where $\nabla 2 V_{i}$ - laplacian $V_{i} ; v=\mu / \rho$ - kinematic coefficient of viscosity; $\mu$ - absolute viscosity coefficient.

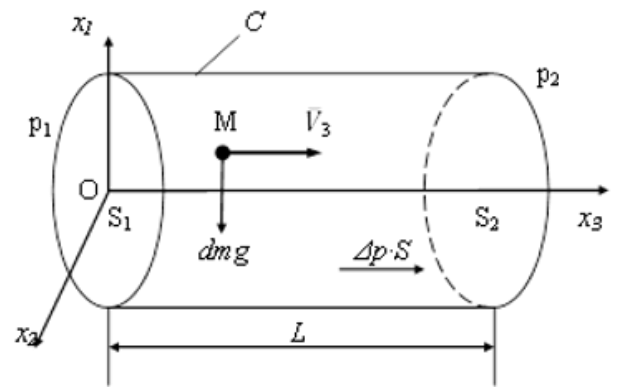

Fig. 3. Schematic diagram of the laminar motion in horizontal of the cylinder bore: $\mathrm{d}$ Tg - elementary weight of the fluid particle $\mathrm{M} ; \mathrm{S} \_\mathrm{i}-$ sectional area $\mathrm{S} \_1=\mathrm{S} \_2=\mathrm{S}$; $\Delta \mathrm{p}=\mathrm{p} 1$ - p2 - differential head; $\mathrm{p} \_1, \mathrm{p} \_2-\overline{\text { high }}$ and low pressure zones consequently; $\mathrm{L}-\overline{\text { line calculated length }}$

Further we will review the fluid motion in the setting zone 2 of the effluents treatment technology (see pic. 1). Fluid motion in a cylinder (pic. 3) is directed along an axis $\mathrm{Ox} 3$, in such a way that from the three speed components (V1, V2, V3) only one remains V3 $=\mathrm{V} 3(\mathrm{x} 1$, $\mathrm{x} 2$ ), and other two are equal to zero. Projections of the volume force on the axis $\mathrm{x} 2$ and $\mathrm{x} 3$ are equal to zero (F2 $=F 3=0), F 1=g$. Then Navier-Stokes equations (3), take the form of:

$$
\begin{gathered}
0=g-\frac{1}{\rho} \frac{\partial p}{\partial x_{1}} ; 0=-\frac{1}{\rho} \frac{\partial p}{\partial x_{2}} \\
\frac{d V_{3}}{d t}=-\frac{1}{\rho} \frac{\partial p}{\partial x_{3}}+v\left(\frac{\partial^{2} V_{3}}{\partial x_{1}^{2}}+\frac{\partial^{2} V_{3}}{\partial x_{2}^{2}}+\frac{\partial^{2} V_{3}}{\partial x_{3}^{2}}\right), \\
\frac{\partial V_{3}}{\partial x_{3}}=0,
\end{gathered}
$$

which we will solve under boundary conditions.

Let us review the third equation of the system (5):

$$
\frac{d V_{3}}{d t}-=-\frac{1}{\rho} \frac{\partial p}{\partial x_{3}}+v\left(\frac{\partial^{2} V_{3}}{\partial x_{1}^{2}}+\frac{\partial^{2} V_{3}}{\partial x_{2}^{2}}\right)
$$

If in the general case

$$
-\frac{\partial p}{\partial x_{3}}=f(t)
$$

Then the differential equation of the nonstationary fluid motion on the basis of (3)-(7) in the culvert barrel of the round cross-section we will write as following

$$
\frac{d V_{3}}{d t}=\frac{1}{\rho} f(t)+v \nabla^{2} V_{3}
$$

Streamline fluid motion in the setting zone canal take place under the forces caused by the differential head $f(t)=\frac{\Delta p}{L}=\frac{p_{1}-p_{2}}{L}=$ const and the forces of liquid friction $R=-v \nabla^{2} V_{3}$, caused by the availability of liquid resistance in the liquid fluid.
Inclusive of round cross-section of the fluid supply system trains $\frac{\partial^{2} V_{3}}{\partial x_{1}^{2}}=\frac{\partial^{2} V_{3}}{\partial x_{2}^{2}}$ we will rewrite the equation (8) as following

$$
\frac{d V_{3}}{d t} \rho=-\frac{\partial p}{\partial x_{3}}+2 \mu \frac{\partial^{2} V_{3}}{\partial x_{1}^{2}}
$$

Fluid velocity profile in the canal of the pipe is presented in broad strokes on the pic.4. In the main part of the flow the longitudinal velocity V3 will coincide in pressure and speed in the flow on the cloak of the boundary layer. Then the speed decrease to the zero value is presented.

It is evident that shearing flow defining the appearance of the shift voltage p23, p13, exists only in the interface zone, the mat thickness of which is equal to $\mathrm{A}$. Instantaneous speed of the fluid flow in the boundary layer $v 3$, at that $0<v 3<\mathrm{V}_{3}$.

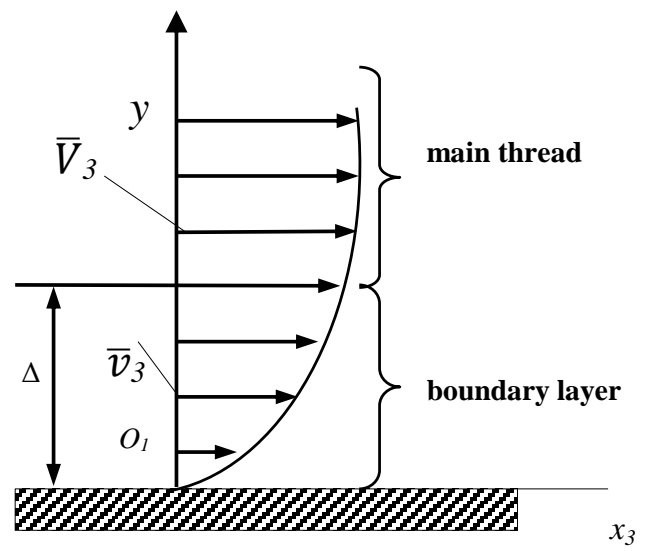

Fig. 4. Fluid velocity profile in the boundary layer: $v_{3}$, V3 - speed in boundary and main fluid flow; A - height of the boundary layer

Boundary layer is understood to be a flow range thin in the direction transverse to the flow, where as distinct from the main flow surrounding it the motion is characterized by the concentrated in these areas violent changes of the speed $v_{3}$ (velocitudionous boundary layer) [11-15].

We will review the fluid flow in the boundary layer in the coordinates y $O_{1} x_{3}$, at that variable coordinate $\mathrm{y}=\mathrm{r}$ $x_{1}$, where $\mathrm{r}$ - radius of the pipe installation canal.

The main condition of appearance of the velocitudionous boundary layers is low fluid velocity, more exactly the great significance of the Reynolds stream numbers Re, which is below that critical value, whereby the flow pattern in the boundary layer becomes turbulent $(\operatorname{Re}>2300)[8,9]$.

The number $\mathrm{Re}$ is a similarity criterion and is determined from the formula

$$
\operatorname{Re}=\frac{U_{0} L_{0}}{v}
$$

where $v$ - coefficient of kinematic viscosity; $L_{0}, U_{0}$ the scale of the longitudinal stretch and velocity from the 
relevant transverse stretch and velocity $\Delta$ and V3. The bore $L_{O}$ diameter can be taken to be the dimension.

As a case in point we will review the pipeline section with the following system parameters: $V_{3}=0,01 \mathrm{~m} / \mathrm{s} ; \mathrm{d}=$ $0,1 \mathrm{~m} ; \boldsymbol{V}$ fluid $=1,004-10-6 \mathrm{~m} 2 / \mathrm{s}$, we receive:

$$
\operatorname{Re}=\frac{0,001}{1,004 \cdot 10^{-6}}=996
$$

The found valuation $\operatorname{Re}=996$ is smaller than the lowest rejection Reynolds number which is approximately equal to Rекр $=2300$, that is why the flow remains its stable laminar form.

As it is known from the [8], the law of variation of the boundary layer thickness ratio is inversely related to the square root of Reynolds number of the flow:

$$
\frac{\Delta}{d}=O\left(\frac{1}{\sqrt{R e}}\right)
$$

where $\mathrm{O}$ - symbol of modification procedure of the intensity with the increase of the Reynolds number.

From the equation (11) we define that $\Delta=3,17 \cdot 10^{-3}$

In line with (9) the speed projection V3 on the axis y (see pic. 4) is written as

$$
\frac{d V_{3}}{d y}=-\frac{d V_{3}}{d x_{1}} ; \frac{d^{2} V_{3}}{d y^{2}}=-\frac{d^{2} V_{3}}{d x_{1}^{2}}
$$

The velocity profile module in the boundary layer of the fluid is in cusp form and is described by the equation

$$
v_{3}=\frac{V_{3}}{\Delta^{2}} y^{2}
$$

where $\Delta$ - is the height of the fluid boundary layer; V3 - speed in the main flow.

It is obvious that if $\mathrm{y}=0, v_{3}=0$; and $\mathrm{y}=\Delta, v_{3}=\mathrm{V} 3$.

The corresponded velocity derivatives and derivative of order two in the plane

$$
\frac{\partial v_{3}}{\partial y}=\frac{2 V_{3}}{\Delta^{2}} y ; \frac{\partial^{2} v_{3}}{\partial y^{2}}=\frac{2 V_{3}}{\Delta^{2}}
$$

Applying (14) to (11), we will receive:

$$
\frac{d V_{3}}{d t} \rho=f(t)-4 \mu \frac{V_{3}}{\Delta^{2}}
$$

Augend of the second member of equation (15) constitute force of the liquid friction $\mathrm{R}=2 \mathrm{nV}$, where $2 \mathrm{n}=\frac{4 \mu}{\rho \Delta^{2}}$, or $n=\frac{2 \mu}{\Delta^{2} \rho}=\frac{2 v}{\Delta^{2}}$.

\subsection{Modeling process of the pollution particle motion in the fluid stream}

As the analytical model of the particle motion in the fluid flow we will take the model shown in the pic.5. The particle of mass $\mathrm{T}$ is in motion in the fluid flow under the action of three forces [16-17]: particle mass $\mathrm{G}=\mathrm{mg}$, force modeling the interaction of particles with the fluid $\bar{R}$, of the buoyancy force $\bar{F}$. Longitudinal movement of the fluid flow with velocity $\bar{V}$ e will be translational and in line with the indicted fluid model in the boundary layer where $\overline{v_{3}}=\bar{V}$ e where y $>\Delta$ and $v_{3}=\frac{V_{3}}{\Delta^{2}} y^{2}$ where $\mathrm{y}<\Delta$, vertical particle motion due to gravity $\overline{V_{r}}$ - is relative. Absolute velocity of the particle motion Va will be found as vector sum of the relative and drift speed:

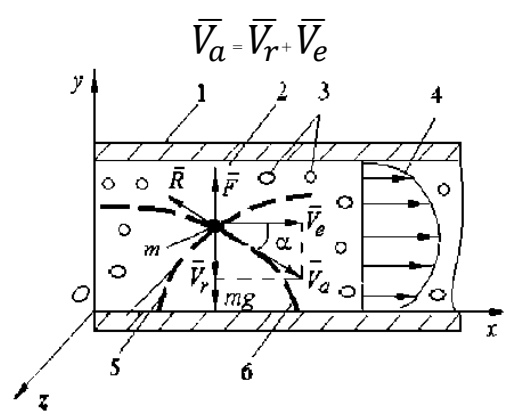

Fig. 5. Analytical model of the pollution particle motion: 1 - condensed model of the cylindrical ducting canal; 2 - fluid flow; 3 - pollution paricles; 4 - epur velocity diagram of the fluid flow;5, 6 - path of particle while impaction and floatation consequently

Let particle motion in the fluid flow take place in a path 6 (see pic.5).

For recording of the particle motion equation we will use the principal law of dynamics:

$$
\overline{\mathrm{a}} \bar{G} \cdot \bar{R} \bar{F}
$$

$\bar{\imath} \ddot{x}+\bar{\jmath} \ddot{y}+\bar{k} \ddot{z}$, taking into consideration that $\mathrm{a}=\bar{l}, \bar{J}$ , $\bar{k}$ where unit vector of the system of coordinates; $\ddot{x}, \ddot{y}$, $\ddot{z}$ - absolute acceleration projection on the axis $\mathrm{x}, \mathrm{y}, \mathrm{z}$.

In our situation for the plane frame $\mathrm{z}=0$ and particle motion is described by the system of two differential equations:

$$
\left\{\begin{array}{l}
m \ddot{x}=\sum \bar{F}_{i x}^{e} \\
m \ddot{y}=\sum \bar{F}_{i y}^{e}
\end{array}\right.
$$

where $\sum \bar{F}_{i x}^{e}, \sum \bar{F}_{i y}^{e}$ - sum of forces, impacting the particle in projection on the axis $\mathrm{x}$ and $\mathrm{y}$ consequently.

Interparticle force with the fluid is defined by the formula

$$
\bar{R}=-\eta \bar{V}_{r}=-\eta\left(\bar{V}_{a}-\bar{V}_{e}\right)
$$

and its projection is equal to:

$$
\left\{\begin{array}{l}
R_{x}=\eta(V-\dot{x}) \\
R_{y}=-\eta \dot{y}
\end{array}\right.
$$

where $\eta$ - interaction coefficient of the particle with the fluid, depending on the particle diameter and shape; $\dot{\mathrm{X}}=V_{a x}, \dot{\mathrm{y}}=$ Vay - absolute velocity projection on the axis $\mathrm{x}$ and $\mathrm{y} ; \mathrm{V}=\mathrm{Vex}$ - transfer velocity projection on the axis $\mathrm{x}, \mathrm{Vey}=0$.

Buoyancy force $\mathrm{F}$ projection on the axis $\mathrm{y}$ will be defined as:

$$
\left\{\begin{array}{l}
F_{y}=\rho g v=\gamma v \\
F_{x}=0
\end{array}\right.
$$

where $\rho, g, v$ - consequently liquid density, intensity of gravity, particle volume; $\gamma$ - relative density of the particle material.

As the result the equation (18) in respect of (20), (21) will become:

$$
\left\{\begin{array}{l}
m \ddot{x}=\eta(V-\dot{x}) \\
m \ddot{y}=\gamma v-m g-\eta \dot{y} .
\end{array}\right.
$$

In accordance with pic. 5 three options of the particle behavior are possible: impaction $(\mathrm{Tg}>\gamma \nu)$, quiescent 
condition $(\mathrm{Tg}=\boldsymbol{\gamma} \boldsymbol{\nu})$ and flotation $(\mathrm{Tg}<\boldsymbol{\gamma})$. Let's consider the special case when $\mathrm{tg}>\gamma \nu$ and $\mathrm{V}=$ const. For this we will transform the equation (22), dividing it term by on $\mathrm{T}$, then: $\bar{\eta}=\frac{\eta}{m}$, and imply $\gamma \nu-\mathrm{Tg}={ }_{\mathrm{T}} \bar{g}$, as the result we will receive:

$$
\left\{\begin{array}{l}
\ddot{x}=\bar{\eta}(V-\dot{x}) \\
\ddot{y}=-\bar{g}-\bar{\eta} \dot{y} .
\end{array}\right.
$$

For the solving of simultaneous equations (23) we will input the substituting:

$$
(V-\dot{x})=-\dot{\bar{x}} ; \frac{\bar{g}}{\bar{\eta}}+\dot{y}=\dot{\bar{y}}
$$

Then considering (24) the simultaneous equations (23) will be as following:

$$
\left\{\begin{array}{l}
\ddot{\bar{x}}=-\bar{\eta} \dot{\bar{x}} \\
\ddot{\bar{y}}=-\bar{\eta} \dot{\bar{y}}
\end{array}\right.
$$

Integrating the equation (25) by dividing of the variables for the case $\mathrm{V}=$ const for the initial data while $\mathrm{t}=0, \dot{\bar{x}}=\mathrm{V} 0 \mathrm{x}, \dot{\bar{y}}=\mathrm{V} 0 \mathrm{y}$, we will receive:

$$
\left\{\begin{array}{l}
\dot{\bar{x}}=V_{0 x} \cdot e^{-\bar{\eta} t} \\
\dot{\bar{y}}=V_{0 y} \cdot e^{-\bar{\eta} t}
\end{array}\right.
$$

We will define $V_{0 x}$ and $V_{0 y}$ from the following inferences: let in the beginning of zone II the impaction has following initial data (while $\mathrm{t}=0$ ):

$$
\begin{gathered}
\mathrm{x}=0, \mathrm{y}=y_{0} \text {, when } 0<y_{0}<\mathrm{d} \\
\dot{\mathrm{x}}=\dot{x}_{0}, \dot{\mathrm{y}}=\dot{y}_{0} \text {, when }-\dot{y}_{0}<\dot{\mathrm{y}}<\dot{y}_{0} .
\end{gathered}
$$

Then

$$
V_{0 x}=\dot{x_{0}}-\mathrm{V}, V_{0 y}=\frac{\bar{g}}{\bar{\eta}}+\dot{y}_{0}
$$

Plugging (27) into (26), considering (24) we will receive:

$$
\left\{\begin{array}{l}
\dot{\mathrm{x}}=V+\left(\mathrm{x}_{0}-V\right) \cdot \mathrm{e}^{-\overline{\eta \mathrm{t}}} \\
\dot{\mathrm{y}}=\left(\frac{\overline{\mathrm{g}}}{\bar{\eta}}+\dot{\mathrm{y}}_{0}\right) \cdot \mathrm{e}^{-\overline{\eta \mathrm{t}}}+\frac{\overline{\mathrm{g}}}{\bar{\eta}}
\end{array}\right.
$$

Integrating (28) over the time we will receive the parametric equations, determining the direction of the particles motion in the fluid:

$$
\left\{\begin{array}{l}
x=V t+\left(\dot{x}_{0}-V\right) \frac{1}{-\bar{\eta}} e^{-\eta t}+C_{3} ; \\
y=\left(\frac{\bar{g}}{\bar{\eta}}+\dot{y}_{0}\right) \frac{1}{-\bar{\eta}} e^{-\bar{\eta} \mathrm{t}}+\frac{\bar{g}}{\bar{\eta}} t+C_{4} .
\end{array}\right.
$$

Plugging the initial data into (29), we will receive:

$$
C_{3}=\frac{\dot{x}_{0}-V}{\bar{\eta}} ; C_{4}=-\left(\frac{\bar{g}+\bar{\eta} \dot{y}_{0}}{\bar{\eta}^{2}}+y_{0}\right)
$$

Analysis of this formula shows that the distance the particle will transfer to depends on initial fluid speed and particles coordinates, interaction coefficient of particle with the fluid and angle between vectors of the speed and acceleration. Thus changing these parameters the process of the impaction of particles can be managed, in other words the manageable cleaning out of the water from the particulate matter can be realized.

For defining of the empirical coefficient of interaction of particles with the fluid $\eta$ there were performed the researches, which made possible to find out the dependence on the intensity $\eta$ of the average grain diameter of the particle $\mathrm{d}$ [18-21]. Approximation grouping together the viscous resistance coefficient $\mu$ and particle diameter becomes,

$$
\eta=\eta_{0}+\eta_{1} d^{2}
$$

where $\eta_{0}=10-7 \mathrm{H} \cdot \mathrm{s} / \mathrm{m}$ - for the particle diameter $\mathrm{d}=$ $0,1 \mathrm{~mm} ; \eta_{1}=10-6 \mathrm{H} \cdot \mathrm{s} / \mathrm{m}-$ for the particle diameter $\mathrm{d}=$ $2 \mathrm{~mm}$.

Experiments proved that the interaction coefficient of the particle with the fluid $\eta$ depends on the particles size (pic.6,a) and the distance the particle transfer to depends on the flow speed and viscous interaction fraction of the particle with fluid (pic. 6, a). Knowing the flow speed the area of impaction of particles and formation of the hard deposits can be defined. Thus there is the critical speed of the flow during which the equipment system will be plugged by hard deposits.

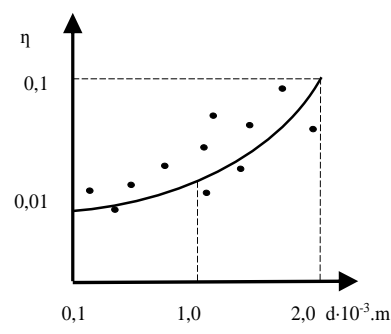

a)

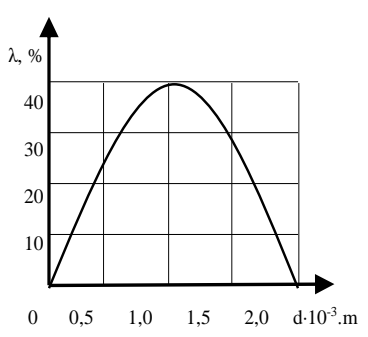

b)
Fig. 6. Dependence of interaction coefficient of the particle with fluid $\eta$ (a) and particle distribution $\lambda$ from the $d(b)$

Particle diameter distribution can be presented diagrammatically, as shown on pic.6, б. Predetermining a value of particle diameter in accordance with the formula (31) we can determine the interaction coefficient value of particle with fluid $\eta$, further according to formula (29) taking into consideration the formula (30) we can calculate the distance the particle will remove to in the sedimentation process.

\section{Conclusion}

The undertaken researches indicate that there is the limiting velocity whereby the equipment system will be clogged by the hard deposits. From henceforth the main attention will be paid to the velocity profile calculations in different constructions for exposure of the critical flow zones. On the basis of this information the sedimentation process can be managed and constrained tools excluding the silt in the parasite areas of the cleansing system can be implemented.

\section{References}

1. Safarov D.I., Abilov F.A., Lapin F.A. Two part analysis of the pollution level of the lubricatingcooling fluid // AzNIIAzVODGEO. Iss. 20. Baku, (1991). p. 112- 117. 
2. Safarov D.I. Dynamics of the immersion vibrator with the flexible operating device // Vibrational machines and technologies: collection of scientific papers Kursk, (1999). p. 108-113.

3. Jatsun S., Safarov J. Mathematical model of granular material in a vibrating boiling // Layers EUROMECH Colloquium 425. Aberdeen, Scotland, (2001). P. 322-326.

4. Jatsun S., Safarov J., Loktionova O. Mathematical Modeling of Processing of Granular Material in a Vibroboiling Layer // First Intern. Symp. on Microgravity Res. and Appl. in Fhisical Sci. and Biotechnology. Sorrento, Italy, (2000). P. 233-239.

5. Yatsun S.F. Simulation of the Dry Granular Media Behavior on Vibrating Bed // Drying Technology. Ed. F. S. Mu-jumdar. (1991). N 4. P. 1081-1089.

6. Safarov D.I., Abilov F.A., Processing of the lubricating-cooling effluent waters for the purpose of their reuse // Chemistry and technology of the water. Kiev, (1990). T. 12, № 12. p. 1111-1114.

7. Safarov D.I., Abilov F.A., Lapin F.A. Automatization of cleaning out and preparation of the lubricating-cooling fluids for their multiply utilization // The problems and methods of improvement of the water-supply system and water removal in large cities: collection of scientific papers. Baku: AzNIIAzVODGEO, (1989). p. 31-32.

8. Loycsanskiy L.G. Fluid mechanics. M.: Nauka, 1987.840 p.

9. Fluid mechanics. Selected / under the general editorship of Krayko A.N. M.: Fizmatlit, (2003). $752 \mathrm{p}$.

10. Yacsun S.F., Yemelyanova O.V., Politov E.N. Simulation of fluid motion in the micrometrical dispensing mechanism //Journal of the Samara scientific centre RAN. (2009). 11, №5(2). p. 373-377.

11. Safarov D.I. Sewage treatment system realized on the basis of filter/coalescer. / Journal of HEIs machine industry. -M.: (2005), №9. - p.63-70.

12. Safarov D.I. Sedimentation process modeling of the solid particles in the sewage stream. / Vibration2010. Manageable vibration technologies and machines: collection of scientific papers - Kursk: Kursk state technology university (2010), P.1. p.39-46.

13. Safarov D.I. Simulation of the dynamic of the fluxion process of the feed sewage disposal in the filter/coalesce / Journal of the Pedagogy University. -Baku: Azerbaijan State Pedagogy University (2010), №2. -p.36-41.

14. Yacsun S.F., Mishenko V.Y., Safarov D.I. Implementation of the vibrational mechatronic modules for the intensification of the mixing process of the liquid fluid. I Automation of production processes in machine building and instrument engineering. Sciences.-tech. collect. /National University "Lviv Polytechnic". -Lviv: Publishing house of National University of "Lviv Polytechnic". (2006) 40. -S. 306-309.

15. Safarov D.I., Zeynalova N.S., Mammadov S.A. Mathematical model construction of the effluents treatment process at the machine builders. /Journal of Pedagogy University. -Baku: Azerbaijan State Pedagogy University. (2008), №6. -p.44-47.

16. Politov E.N., Yacsun S.F., Safarov D.I. Scientific instrument for flow characteristics of the heterogeneous environment. / Journal of Samara scientific Centre of the Russia Sciences Academy. Samara: (2012), 14, №4(5),-p.1423-1425.

17. Safarov D.I., Yemelyanova O.V., Loktionova O.G. Modeling process of the pollution particle motion in the fluid stream. / Journal of the South-West State University, - Kursk: South-West State University, (2012). - №4(43). P.2. -p.225-227.

18. Khuzhakulov T., Burieva M., Tursunaliev J. Waste water treatment of industrial enterprises by modeling polyaniline sorption materials//Colloquium-journal. 3-2 (55), (2020). C.113-116 (in Russian).

19. Varaksin A. Yu. Two-phase flows with solid particles, droplets and bubbles: problems and research results//Thermophysics of high temperatures. 58, No. 4. (2020). C. 646-669 (in Russian).

20. Liu, L.T., Wang, C.L.; Yu, X.Y.; Shi, J.K.; Li, Y.; Chen, X.M.; Zhou, W.H. Study of nano particle stripping and composition inspection on wafer surface // Acta Physica Sinica. Том: 69 Выпуск: 16, (2020).

21. Desogus, F; Carta, R. Experimental Determination of the Particle Dynamics into a Rotating Tube // PRES2016: 19th International Conference On Process Integration, Modeling And Optimization For Energy Savings And Pollution Reduction. Tом: 52, 2016, pp. 1123-1128. 